\title{
Ellagic acid inhibits high glucose-induced injury in rat mesangial cells via the PI3K/Akt/FOXO3a signaling pathway
}

\author{
WEI LIN ${ }^{1 *}$, GUOJIAN LIU ${ }^{2 *}$, XIAOWEN KANG ${ }^{3}$, PING GUO $^{4}$, YU SHANG $^{2}$, RUOMEI DU $^{2}$, \\ XIYUE WANG ${ }^{2}$, LITING CHEN ${ }^{2}$, RUI YUE ${ }^{2}$, FANWU KONG ${ }^{2}$ and QIHAN ZHU ${ }^{5}$ \\ ${ }^{1}$ Department of General Medicine, The First Affiliated Hospital of Wenzhou Medical University, Wenzhou, Zhejiang 325000; \\ Departments of ${ }^{2}$ Nephrology and ${ }^{3}$ Respiration, The Second Affiliated Hospital of Harbin Medical University, Harbin, \\ Heilongjiang 150086; ${ }^{4}$ Laboratory Department, Heilongjiang Academy of Traditional Chinese Medicine, \\ Harbin, Heilongjiang 150036; ${ }^{5}$ Department of Endocrinology, The First Affiliated Hospital of \\ Wenzhou Medical University, Wenzhou, Zhejiang 325000, P.R. China
}

Received October 18, 2020; Accepted June 16, 2021

DOI: 10.3892/etm.2021.10449

\begin{abstract}
The pathological damage of mesangial cells serves an important role in the occurrence and development of diabetic nephropathy.Ellagicacidhas beenreported topossess antioxidant, antitumor, antiviral and anti-inflammatory properties in several diseases, but the roles of ellagic acid in diabetic nephropathy are unclear. The main aim of the present study was to investigate the effect of ellagic acid on high glucose-induced mesangial cell damage. The results revealed that high glucose could induce the hyperproliferation of mesangial cells, decrease the activity of superoxide dismutase, increase the malondialdehyde content, the level of reactive oxygen species, the secretion of inflammatory factors (TNF- $\alpha$, IL-1 $\beta$ and IL-6) and the synthesis of extracellular matrix (Fibronectin, MMP-9 and TIMP-1) and activate the PI3K/Akt/FOXO3a signaling pathway. Ellagic acid could attenuate the injury of mesangial cells induced by high glucose in a concentration-dependent manner and its effect was consistent with that of a PI3K inhibitor (LY294002). Moreover, a PI3K agonist (740Y-P) reversed the protective effect of ellagic acid on mesangial cells induced by high glucose. In conclusion, ellagic acid protected mesangial cells from high glucose-induced injury in a concentration-dependent manner. The mechanism may be associated with ellagic acid inhibiting the activation of
\end{abstract}

Correspondence to: Dr Qihan Zhu, Department of Endocrinology, The First Affiliated Hospital of Wenzhou Medical University, 2 Fuxue Lane, Lucheng, Wenzhou, Zhejiang 325000, P.R. China E-mail: seno6257@21cn.com

Dr Fanwu Kong, Department of Nephrology, The Second Affiliated Hospital of Harbin Medical University, 246 Xuefu Road, Harbin, Heilongjiang 150086, P.R. China

E-mail: kongfanwu067@126.com

${ }^{*}$ Contributed equally

Key words: ellagic acid, mesangial cells, diabetic nephropathy, $\mathrm{PI} 3 \mathrm{~K} / \mathrm{Akt}$, FOXO3a the PI3K/Akt signaling pathway and reducing the expression levels of downstream transcription factor FOXO3a.

\section{Introduction}

Diabetic nephropathy (DN) is a common complication of diabetes and is the main cause of end-stage renal disease in China and worldwide (1). DN is characterized by persistent proteinuria, increase of protein glomerular filtration and abnormal renal function. The early pathological changes include the accumulation of extracellular matrix (ECM) components and renal hypertrophy and the late pathological changes comprise the development of glomerulosclerosis and tubulointerstitial fibrosis (2-4). The abnormal proliferation of mesangial cells (MCs) may partially account for the increase of mesangial matrix and glomerulosclerosis $(5,6)$. Therefore, reducing the damage of mesangial cells may serve a notable role during the treatment of $\mathrm{DN}$.

A number of studies have indicated that oxidative stress plays a considerable role in the occurrence and development of DN $(6,7)$. Recent studies have demonstrated that the stimulation with various factors, such as high glucose (HG), hydrogen peroxide, angiotensin II, aldosterone and inflammation leads to an increase of oxidative stress in MCs, thereby promoting the occurrence of glomerulosclerosis $(8,9)$. Therefore, decreasing or terminating oxidative stress injury of glomerular mesangial cells could improve early diabetic nephropathy. Hyperglycemia induces the increase of reactive oxygen species (ROS) production in glomerular mesangial cells, enhances the activation of transcriptional activator protein-1 (AP-1) and NF- $\kappa \mathrm{B}$ and promotes the expression of inflammatory factors, such as intercellular adhesion molecule and monocyte chemoattractant protein-1, thereby aggravating the local inflammatory response and oxidative stress (10). It was hypothesized that angiotensin is an important mediator of oxidative stress and angiotensin receptor blockers could reduce the level of oxidative stress in patients with diabetes and alleviate renal injury (11).

Ellagic acid (EA) is a natural compound that exists in various soft fruits, nuts and other plant tissues. It has been demonstrated that EA has antioxidant, anticancer, anti-atherosclerotic and 
anti-inflammatory effects in multiple diseases, including ulcerative colitis, colon carcinoma, hepatopathy, Alzheimer's disease and Diabetes (12-14). EA has been indicated to inhibit attenuated renal dysfunction and oxidative stress in high fat diet/low dose streptozotocin (HFD/STZ)-induced type 2 diabetic Wistar albino rats by inhibiting the renal NF- $\kappa \mathrm{B}$ activation (15). Furthermore, EA can reduce ROS production induced by HG in human aortic endothelial cells by promoting ERK1/2 phosphorylation and increasing the expression of NADPH oxidase 4 (NOX4). However, whether EA could improve the damage of glomerular mesangial cells induced by $\mathrm{HG}$ and the associated mechanism is still unclear. The present study investigated the protective effect of EA on the injury of glomerular mesangial cells induced by HG and further explored the implicated molecular mechanism.

\section{Materials and methods}

Cell culture and treatment. EA, D-glucose and the PI3K inhibitor (LY294002) were purchased from Sigma-Aldrich; Merck KGaA. Rat MCs (HBZY-1) were purchased from the China Center for Type Culture Collection, cat. no. GDC0124 (http://cctcc.whu. edu.cn/portal/no_center/cell_detail/id/101.html). MCs were cultured in low glucose or HG DMEM containing $10 \%$ fetal bovine serum (both Gibco; Thermo Fisher Scientific, Inc.). The concentrations of D-glucose and streptomycin/penicillin (Gibco; Thermo Fisher Scientific, Inc.) were $5.5 \mathrm{mM}$ (or $30 \mathrm{mM}$ ) and $100 \mathrm{U} / \mathrm{ml}$, respectively. Cells were incubated in a constant temperature incubator with $5 \% \mathrm{CO}_{2}$ at $37^{\circ} \mathrm{C}$. When the cells grew to $80 \%$ confluency, they were treated as follows: i) Normal control group (NC), MCs were cultured in DMEM with $5.5 \mathrm{mM}$ glucose; ii) HG group, MCs were cultured in DMEM with $30 \mathrm{mM}$ glucose; iii) $\mathrm{HG}$ and low concentration EA intervention group (L-EA), MCs were cultured in DMEM with $30 \mathrm{mM}$ glucose and treated with $10 \mu \mathrm{M}$ EA; iv) HG and medium concentration EA intervention group (M-EA), MCs were cultured in DMEM medium with $30 \mathrm{mM}$ glucose and treated with $20 \mu \mathrm{M} \mathrm{EA}$; v) HG and high concentration EA intervention group (H-EA), MCs were cultured in DMEM with $30 \mathrm{mM}$ glucose and treated with $40 \mu \mathrm{M}$ EA; and vi) HG and PI3K inhibitor (LY294002) group, MCs were cultured in DMEM with $30 \mathrm{mM}$ glucose and treated with $20 \mu \mathrm{M}$ LY294002. Overall, MCs were cultured in DMEM with $5.5 \mathrm{mM}$ glucose or DMEM with $30 \mathrm{mM}$ glucose plus EA $(10,20$ or $40 \mu \mathrm{M})$ or DMEM with $30 \mathrm{mM}$ glucose and $20 \mu \mathrm{M}$ LY294002 at $37^{\circ} \mathrm{C}$ for $24 \mathrm{~h}(16,17)$.

To detect the cytotoxic effect of EA on MCs, different concentrations of $\mathrm{EA}(0,1,5,10,20,40$ or $80 \mu \mathrm{M})$ or $20 \mu \mathrm{M}$ LY294002 were incubated with MCs under low glucose conditions at $37^{\circ} \mathrm{C}$ for $24 \mathrm{~h}$.

To examine whether EA mediated protection of $\mathrm{MCs}$ following $\mathrm{HG}$ treatment via the PI3K/Akt signaling pathway, HG-stimulated MCs were treated with EA $(40 \mu \mathrm{M})$ and/or the PI3K agonist $740 \mathrm{Y}-\mathrm{P}\left(10 \mu \mathrm{M}\right.$, MedChemExpress) at $37^{\circ} \mathrm{C}$ for $24 \mathrm{~h}(18)$.

MTT assay. At a density of $1 \times 10^{5}$ cells $/ \mathrm{ml}$, MCs in the logarithmic growth phase were inoculated into a 96-well plate $\left(4 \times 10^{3}\right.$ cells/well) and subsequently cultured in a $5 \%$ $\mathrm{CO}_{2}$ incubator at $37^{\circ} \mathrm{C}$ for $24 \mathrm{~h}$. After the cells were treated as aforementioned for $24 \mathrm{~h}, 25 \mu \mathrm{l}$ MTT $(5 \mathrm{mg} / \mathrm{ml})$ solution was added into each well. After incubation at $37^{\circ} \mathrm{C}$ for $4 \mathrm{~h}, 100 \mu \mathrm{l}$ dimethyl sulfoxide was added into each well. After 2 min oscillation of the plate, the absorbance value was measured at $570 \mathrm{~nm}$ using a microplate reader (BioTek Instruments, Inc.).

5-Ethynyl-2'-deoxyuridine (EdU) assay. MCs, treated as aforementioned, were incubated in 96-well plates at $4 \times 10^{3}$ cells/well in a $5 \% \mathrm{CO}_{2}$ incubator at $37^{\circ} \mathrm{C}$ for $24 \mathrm{~h}$. Subsequently, $1 \mathrm{ml} \mathrm{4 \%}$ paraformaldehyde was used for fixation at room temperature for $15 \mathrm{~min}$ and then cells were washed with PBS containing 3\% BSA (Sigma-Aldrich; Merck KGaA). Cells were subsequently incubated with $1 \mathrm{ml} 0.5 \%$ Triton X-100 at room temperature for $20 \mathrm{~min}$. After being washed with PBS containing 3\% BSA, cells in each group were treated with $10 \mu \mathrm{M}$ EdU for $30 \mathrm{~min}$ at room temperature. After washing with PBS, DAPI $(5 \mu \mathrm{g} / \mathrm{ml})$ was added to the cells in the dark for $5 \mathrm{~min}$ at room temperature. The cells were observed under an inverted fluorescence microscope (Leica DMI3000-B; Leica Microsystems $\mathrm{GmbH}$ ) and the number of positive cells was counted from five random fields in three wells using ImageJ $1.48 \mathrm{v}$ software (National Institutes of Health).

Measurement of ROS. The intracellular ROS of MCs were detected using a ROS detection kit (cat. no. S0033S; Beyotime Institute of Biotechnology) according to the manufacturer's instructions. Briefly, 2',7'-dichlorodihydrofluorescein diacetate (DCFH-DA) was diluted with serum-free DMEM at a ratio of $1: 1,000$ to a final concentration of $10 \mu \mathrm{M}$. The cells were collected and suspended in the diluted DCFH-DA at a concentration of $1 \times 10^{8} / \mathrm{ml}$, incubated at $37^{\circ} \mathrm{C}$ for $20 \mathrm{~min}$ and then washed with serum-free DMEM three times. A fluorescence microscope (Leica DMI3000-B; Leica Microsystems GmbH) was used to observe and capture images. The fluorescence intensity was detected using a microplate reader (BioTek Instruments, Inc.), at an excitation wavelength of $488 \mathrm{~nm}$ and an emission wavelength of $525 \mathrm{~nm}$.

Detection of malondialdehyde (MDA) content and superoxide dismutase (SOD) activity. MDA content was detected using a commercial kit (cat. no. S0131S; Beyotime Institute of Biotechnology) and was performed in accordance with the manufacturer's instructions. MCs were lysed for $30 \mathrm{~min}$ on ice using RIPA lysis buffer (Beyotime Institute of Biotechnology) and centrifuged at $12,000 \mathrm{rpm}$ for $10 \mathrm{~min}$. Subsequently, $100 \mu \mathrm{l}$ supernatant was obtained and mixed with $200 \mu \mathrm{l}$ MDA working solution. Following that, the solution was heated at $100^{\circ} \mathrm{C}$ for $15 \mathrm{~min}$, cooled to room temperature and centrifuged at $1,000 \mathrm{x} \mathrm{g}$ for $10 \mathrm{~min}$ at room temperature. Subsequently, $200 \mu \mathrm{l}$ supernatant/MDA mixture was added into 96-well plates and the absorbance value was determined at $532 \mathrm{~nm}$ using a microplate reader (BioTek Instruments, Inc.). SOD activity was detected using total SOD activity detection kit (WST-8 method; cat. no. S0101S; Beyotime Institute of Biotechnology), according to the manufacturer's instructions. After the cells were lysed for $30 \mathrm{~min}$ on ice using SOD sample preparation solution, $160 \mu \mathrm{l}$ WTS-8/enzyme working solution and $20 \mu \mathrm{l}$ reaction working solution were mixed with $20 \mu \mathrm{l}$ supernatant and then incubated at $37^{\circ} \mathrm{C}$ for $30 \mathrm{~min}$. After this incubation, the absorbance value at $450 \mathrm{~nm}$ was detected using a microplate reader. 
A<smiles></smiles>

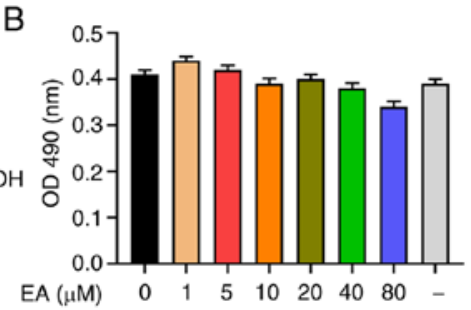

LY294002 ( $\mu$ M)
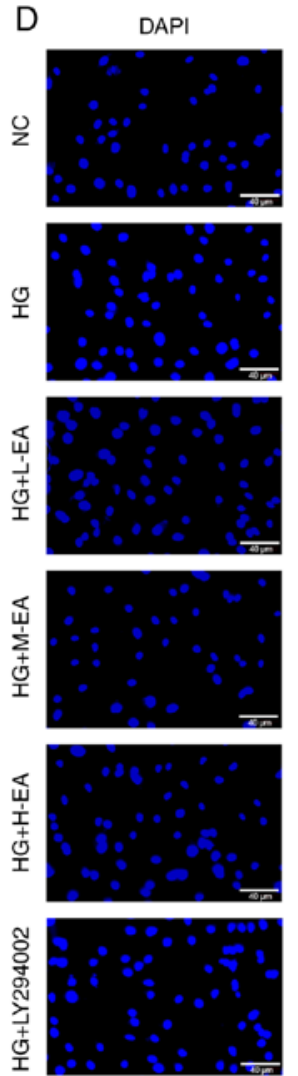
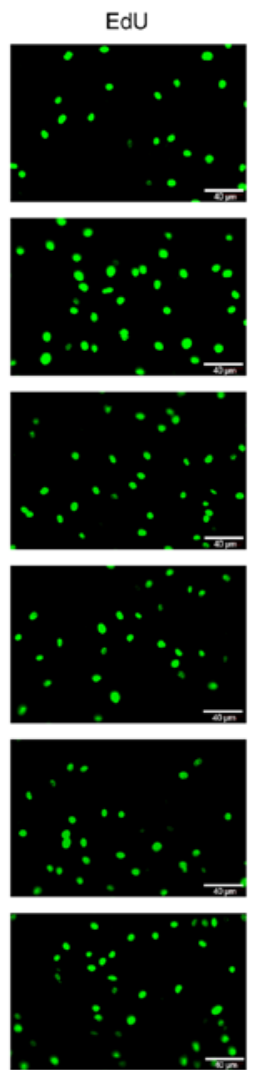
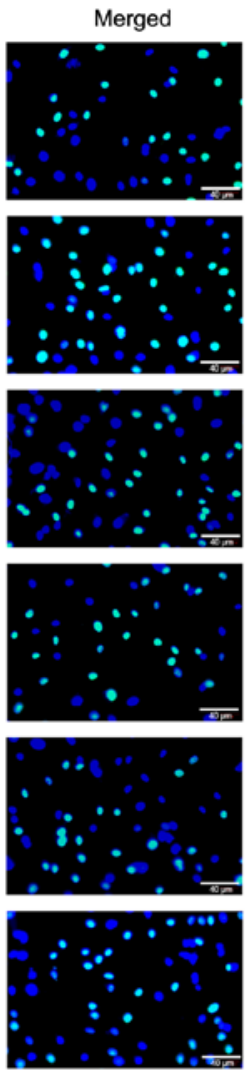

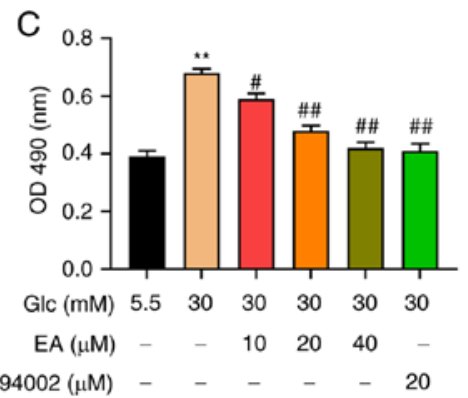

E

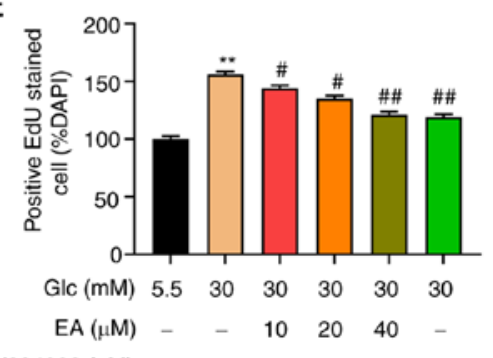

LY294002 $(\mu \mathrm{M}) \quad-\quad-\quad-\quad-20$

Figure 1. EA inhibits the proliferation of MCs induced by HG. (A) Chemical structure of EA. (B) Cytotoxicity of EA (1-80 $\mu$ M) and LY294002 (20 $\mu$ M) was detected via MTT assay in MCs treated with $5.5 \mathrm{mM}$ glucose. (C) Effect of EA (10, 20 and $40 \mu \mathrm{M})$ and LY294002 (20 $\mu \mathrm{M})$ on MC viability was detected using MTT assay. Effect of EA (10, 20 and $40 \mu \mathrm{M})$ and LY294002 $(20 \mu \mathrm{M})$ on MC proliferation was (D) detected by EdU assay and (E) quantified. Scale bar, $40 \mu \mathrm{m}$. ${ }^{* *} \mathrm{P}<0.01$ vs. NC group; ${ }^{*} \mathrm{P}<0.05$ and ${ }^{\# \#} \mathrm{P}<0.01$ vs. HG group. normal control (NC) group refers to $5.5 \mathrm{mM}$ glucose treated cells. EA, ellagic acid; MCs, mesangial cells; OD, optical density; HG, high glucose; H-EA, high concentration EA intervention group; M-EA, medium concentration EA intervention group; L-EA, low concentration EA intervention group; NC, normal control; Glc, glucose; EdU, 5-ethynyl-2'-deoxyuridine.

ELISA. MCs were inoculated into 12-well plates at a density of $9 \times 10^{5}$ cells/well. They were incubated at $37^{\circ} \mathrm{C}$ in $5 \% \mathrm{CO}_{2}$ for $24 \mathrm{~h}$. MCs were treated as aforementioned and cultured at $37^{\circ} \mathrm{C}$ for $24 \mathrm{~h}$. The cell culture medium was collected and centrifuged at $1,000 \mathrm{x} \mathrm{g}$ for $10 \mathrm{~min}$ at $4^{\circ} \mathrm{C}$ and the supernatant was subsequently collected. The levels of IL-6 (cat. no. SR6000B), IL-1 $\beta$ (cat. no. SRLB00), TNF- $\alpha$ (cat. no. SRTA00), fibronectin (FN) (cat. no. ab108850), tissue inhibitor of metalloproteinases 1 (TIMP-1; cat. no. SRTM100) and MMP-9 (cat. no. DY8174-05) were detected using commercial ELISA kits (R\&D Systems, Inc. or Abcam) according to the manufacturer's instructions. Detection was carried out using a microplate reader (BioTek Instruments, Inc.) at $450 \mathrm{~nm}$.

Western blotting. Nuclear proteins were obtained using NE-PER ${ }^{\mathrm{TM}}$ Nuclear and Cytoplasmic Extraction Reagents kit (cat. no. 78833; Thermo Fisher Scientific, Inc.) according to the manufacturer's instructions. Total protein of MCs from each group was extracted using RIPA lysis buffer (Beyotime Institute of Biotechnology) and the protein concentration was measured with BCA protein assay kit (Beyotime Institute of Biotechnology). The same amount of protein $(50 \mu \mathrm{g})$ sample per lane was electrophoresed on a $12 \%$ SDS-PAGE and then transferred onto PVDF membranes. The membranes were blocked using $5 \%$ skimmed milk powder for $1 \mathrm{~h}$ at room temperature. Following blocking, the primary antibodies were added and the membranes were incubated overnight at $4^{\circ} \mathrm{C}$. After being washed three times with TBST containing $0.1 \%$ Tween-20, the membranes were incubated with HRP-conjugated secondary antibodies at room temperature for $2 \mathrm{~h}$. After the membranes were washed three times with TBST, ECL reagent (Bio-Rad Laboratories, Inc.) was used to visualize the bands. Densitometry was performed by ImageJ $1.48 \mathrm{v}$ software (National Institutes of Health). GAPDH was 
used as internal reference. The antibodies were obtained from Abcam as follows: Anti-PI3K (cat. no. ab191606; 1:1,000), anti-phosphorylated (p)-PI3K (cat. no. ab182651; 1:1,000), anti-AKT (cat. no. ab179463; 1:1,000), anti-p-AKT (cat. no. ab81283; 1:1,000), anti-FOXO3a (cat. no. ab109629; 1:1,000), anti-SOD2 (cat. no. ab13533; 1:1,000), anti-p-NF-кB (cat. no. ab76302; 1:1,000), anti-NF-кB (cat. no. ab16502; 1:1,000), anti-lamin B1 (cat. no. ab16048; 1:1,000), anti-GAPDH (cat. no. ab181602; 1:2,000).

Statistical analysis. SPSS v13.0 (SPSS, Inc.) and GraphPad Prism v6.01 software (GraphPad Software, Inc.) was used for statistical analysis and presentation of the data. The data are presented as the mean \pm standard deviation. The comparison between multiple groups was conducted using one-way ANOVA followed by Tukey's post hoc test. All experiments were performed in triplicate. $\mathrm{P}<0.05$ was considered to indicate a statistically significant difference.

\section{Results}

Effects of EA on the viability and proliferation of MCs induced by $H G$. The chemical formula of EA is presented in Fig. 1A. Different concentrations of EA $(0,1,5,10,20,40$ or $80 \mu \mathrm{M})$ or $20 \mu \mathrm{M}$ LY294002 were incubated with MCs under low glucose conditions for $24 \mathrm{~h}$. An MTT assay was used to detect the possible effect of EA toxicity on MCs. The results revealed that the survival rate of MCs exhibited no significant change, indicating that EA in this concentration range had no effect on the viability of MCs (Fig. 1B). Furthermore, the effect of EA on the viability of MCs induced by $\mathrm{HG}$ was examined. The MTT assay results indicated that compared with the NC group, the viability of MCs in the $\mathrm{HG}$ group was significantly increased (Fig. 1C). Compared with the HG group, EA groups $(10,20$ and $40 \mu \mathrm{M})$ and the LY294002 group could significantly inhibit the increase of cell viability induced by $\mathrm{HG}$ and $40 \mu \mathrm{M}$ EA treatment caused the greatest decrease (Fig. 1C).

To detect the effect of EA on MC proliferation, EdU assay was performed. As demonstrated in Fig. 1D and E, the number of positive cells in the $\mathrm{HG}$ group was significantly increased compared with the $\mathrm{NC}$ group, indicating that $\mathrm{HG}$ could significantly increase the proliferation of MCs. Furthermore, compared with the HG group, EA groups (10, 20 and $40 \mu \mathrm{M})$ and the LY294002 group could significantly attenuate the cell hyperproliferation induced by $\mathrm{HG}$ and $40 \mu \mathrm{M}$ EA treatment resulted in the greatest decrease.

Effects of EA on ROS, MDA and SOD in MCs following $H G$ treatment. Following EA treatment, the levels of ROS in MCs were detected using a fluorescence microscope and the levels of SOD and MDA were measured. The content of ROS in MCs was detected using a DCFH-DA probe. The results revealed that compared with the $\mathrm{NC}$ group, the fluorescence intensity of MCs in the HG group was significantly increased, indicating that HG significantly increased the level of ROS (Fig. 2A and B). In addition, compared with the HG group, the fluorescence intensity of MCs in the EA groups $(10,20$ and $40 \mu \mathrm{M})$ and LY294002 group decreased significantly, indicating that ROS level in each group was significantly decreased. Moreover, the fluorescence intensity was attenuated with the increase of EA concentration, which indicated that EA alleviated oxidative stress in a concentration-dependent manner (Fig. 2A and B).

MDA content and SOD activity was detected in MCs cells using commercial kits. The results indicated that the activity of SOD in the HG group was significantly lower compared with the NC group and that the MDA level was higher (Fig. 2C and D). Compared with the HG group, the activity of SOD in the EA groups $(10,20$ and $40 \mu \mathrm{M})$ and the LY294002 group was significantly increased and MDA level was decreased. The effect of EA on MDA content and SOD activity was enhanced with the increase of EA concentration.

Effects of EA on the expression level of inflammatory factors and ECM molecules in MCs induced by HG. In the present study, the levels of FN, MMP-9, TIMP-1, IL-1 $\beta$, IL-6 and TNF- $\alpha$ in MCs were detected by ELISA. The results revealed that the expression levels of IL- 6 , IL- $1 \beta$ and TNF- $\alpha$ were significantly increased in the $\mathrm{HG}$ group, but were inhibited by a dose-dependent EA treatment and LY294002 (Fig. 3A-C). Furthermore, the levels of ECM components were detected. The results demonstrated that compared with the NC group, the FN levels in the HG group were significantly increased, which indicated that $\mathrm{HG}$ resulted in increased ECM synthesis (Fig. 3D). In addition, the levels of MMP-9 and TIMP-1 were significantly increased, but the ratio of MMP-9/TIMP-1 was significantly decreased compared with the NC group, indicating that $\mathrm{HG}$ induced the degradation of ECM in MCs (Fig. 3E-G). Moreover, the increase of FN, MMP-9 and TIMP-1 contents and the decrease of MMP-9/TIMP-1 ratio induced by $\mathrm{HG}$ were partially alleviated by a dose-dependent treatment with EA and LY294002.

Effect of EA on the PI3K/Akt signaling pathway and $p-N F-\kappa B$ and FOXO3a expression levels in the nuclei of MCs following $H G$ treatment. FOXO3a and $\mathrm{NF}-\kappa \mathrm{B}$ are downstream transcription factors of the PI3K/Akt signaling pathway $(19,20)$. It was hypothesized that EA improved the damage of MCs induced by $\mathrm{HG}$ by regulating the PI3K/Akt signaling pathway and the nuclear expression of FOXO3a and NF- $\mathrm{BB}$. The expression levels of PI3K, p-PI3K, Akt, p-Akt, FOXO3, SOD2 and $\mathrm{p}-\mathrm{NF}-\kappa \mathrm{B}$ in $\mathrm{MCs}$ were detected using western blotting. The results revealed that the relative expression levels of $\mathrm{p}-\mathrm{PI} 3 \mathrm{~K}$ and p-Akt in the HG group were significantly higher compared with those in the $\mathrm{NC}$ group, while the relative expression level of SOD2 was significantly decreased (Fig. 4A and C-E). In addition, compared with the $\mathrm{HG}$ group, the relative expression levels of p-PI3K and p-Akt in the EA groups $(10,20$ and $40 \mu \mathrm{M})$ and the LY294002 group were significantly decreased and the relative expression level of SOD2 was significantly increased. The effect of EA was concentration dependent. Moreover, the EA groups $(10,20$ and $40 \mu \mathrm{M})$ and the LY294002 group reduced the level of $\mathrm{p}-\mathrm{NF}-\kappa \mathrm{B}$ and increased the expression of FOXO3a in the nucleus of MCs induced by $\mathrm{HG}$ (Fig. 4B, F and G).

PI3K agonist $740 Y-P$ reverses the effects of $E A$ on the $P I 3 K / A k t$ signaling pathway and the $p-N F-\kappa B$ and FOXO3a expression levels in the nuclei of MCs following HG treatment. To investigate whether EA mediated protection of MCs following HG treatment via regulating the PI3K/Akt signaling pathway, HG-stimulated MCs were treated with EA $(40 \mu \mathrm{M})$ 

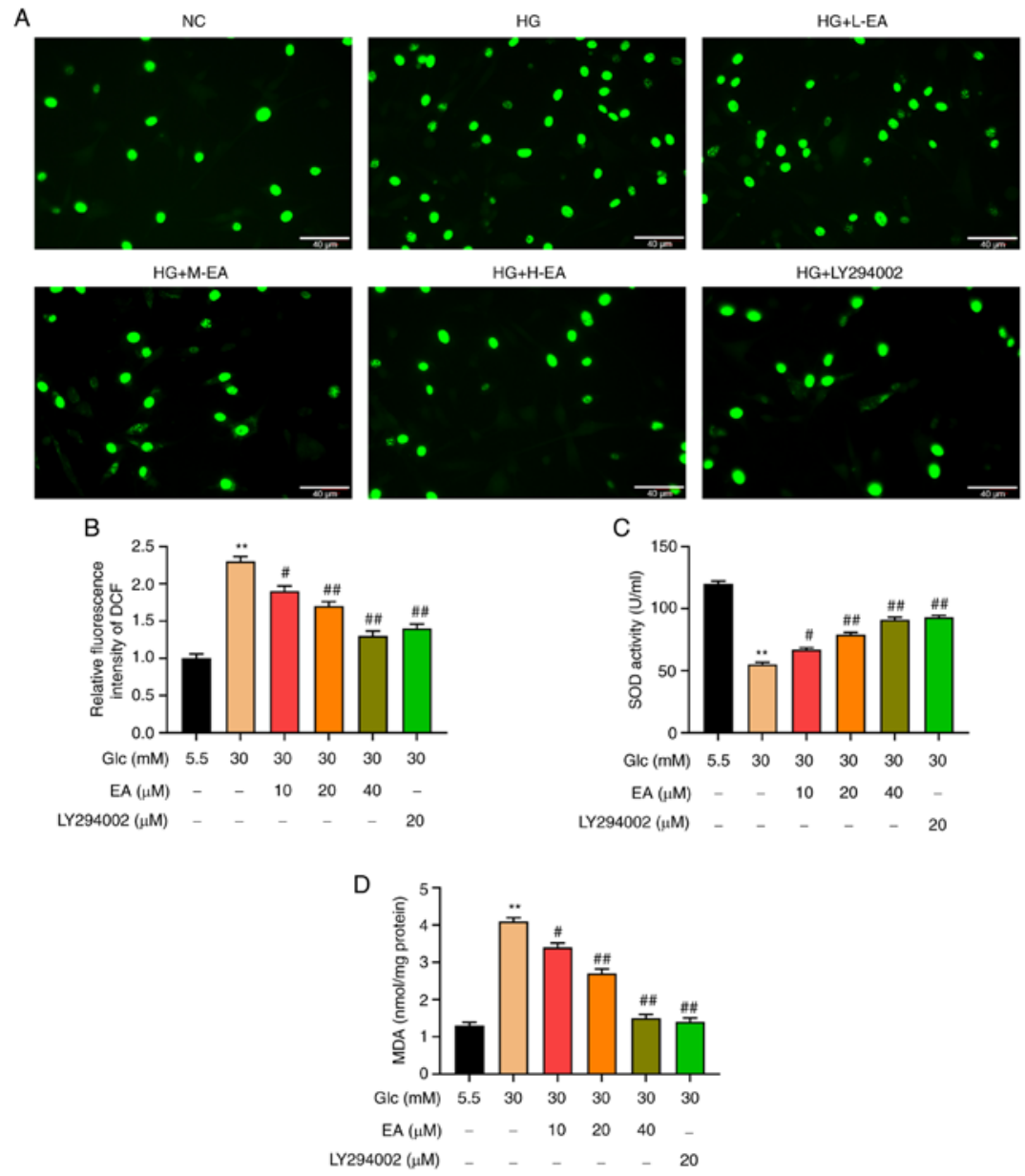

Figure 2. Antioxidant effect of EA on MCs treated with HG. Effect of EA (10,20 and $40 \mu \mathrm{M})$ and LY294002 (20 $\mu \mathrm{M})$ on (A and B) the levels of reactive oxygen species, (C) SOD activity and (D) MDA content in MCs induced by HG (30 mM). Scale bar, $40 \mu \mathrm{m}$. ${ }^{* *} \mathrm{P}<0.01 \mathrm{vs}$. NC group; ${ }^{*} \mathrm{P}<0.05$ and ${ }^{\# \#} \mathrm{P}<0.01 \mathrm{vs}$. HG group $\mathrm{NC}$ group refers to $5.5 \mathrm{mM}$ glucose treated cells. EA, ellagic acid; MCs, mesangial cells; HG, high glucose; H-EA, high concentration EA intervention group; M-EA, medium concentration EA intervention group; L-EA, low concentration EA intervention group; NC, normal control; SOD, superoxide dismutase; MDA, malondialdehyde; DCF, dichlorofluorescein; Glc, glucose.

and/or 740Y-P $(10 \mu \mathrm{M})$ for $24 \mathrm{~h}(18)$. The results revealed that $740 \mathrm{Y}-\mathrm{P}$ further increased intranuclear $\mathrm{p}-\mathrm{NF}-\kappa \mathrm{B}$ levels and the phosphorylation of PI3K and Akt and decreased intranuclear FOXO3a and SOD2 expression levels. However, EA treatment exerted the opposite effect. In addition, 740Y-P could attenuate the effect of EA on the PI3K/Akt signaling pathway, intranuclear $\mathrm{NF}-\kappa \mathrm{B}$, intranuclear FOXO3a and SOD2 expression level (Fig. 5A-G). According to the aforementioned results, PI3K inhibitor alleviated the injury of MCs stimulated by HG. These results suggested that EA attenuated the damage of HG-treated MCs by regulating the PI3K/Akt signaling pathway.

\section{Discussion}

MCs participate in the early pathological changes of DN, such as glomerular hypertrophy and ECM accumulation $(5,21,22)$. Hyperglycemia is the main cause of DN (23). Under normal conditions the number of MCs is relatively stable, but under hyperglycemia abnormal proliferation and ECM production are observed in MCs, which eventually leads to glomerulosclerosis. Therefore, MCs are being mainly investigated in several studies on the pathogenesis of DN $(24,25)$. HG-induced injury markers in MCs include the abnormal proliferation of cells, the increase of ROS and inflammatory factors and the accumulation of ECM (26). The present study used HG to treat rat MCs to establish a DN model in vitro. EA is a type of polyphenol compound, widely used in Chinese herbal medicines, such as sanguisorba officinalis and pomegranate peel $(27,28)$. Numerous studies have demonstrated that EA possesses multiple biological activities in vitro $(14,29,30)$. The present study explored whether EA could prevent the HG-induced injury of MCs and its possible mechanism.

The proliferation of MCs can be abnormal due to various pathophysiological changes and it can affect renal function through multiple pathways. On the one hand, excessive proliferation of MCs can induce cell adhesion factors (intercellular cell adhesion molecule-1), ECM (MMPs, laminin and fibronectin) and other substances (TNF- $\alpha$, IL-1 $\beta$ and IL-6) to accumulate in the mesangial region. On the other, excessive proliferation of MCs can promote the release and secretion of inflammatory mediators, cytokines and growth factors and accelerate the deterioration of renal function (31-33). A recent study has indicated that EA inhibited breast cancer cell proliferation and increased apoptosis by downregulating CDK6 expression (34). In addition, EA has been demonstrated to attenuate prostate carcinoma PC3 cell proliferation and viability via activating caspases and inducing apoptosis (35). The results of the present study were consistent with these 


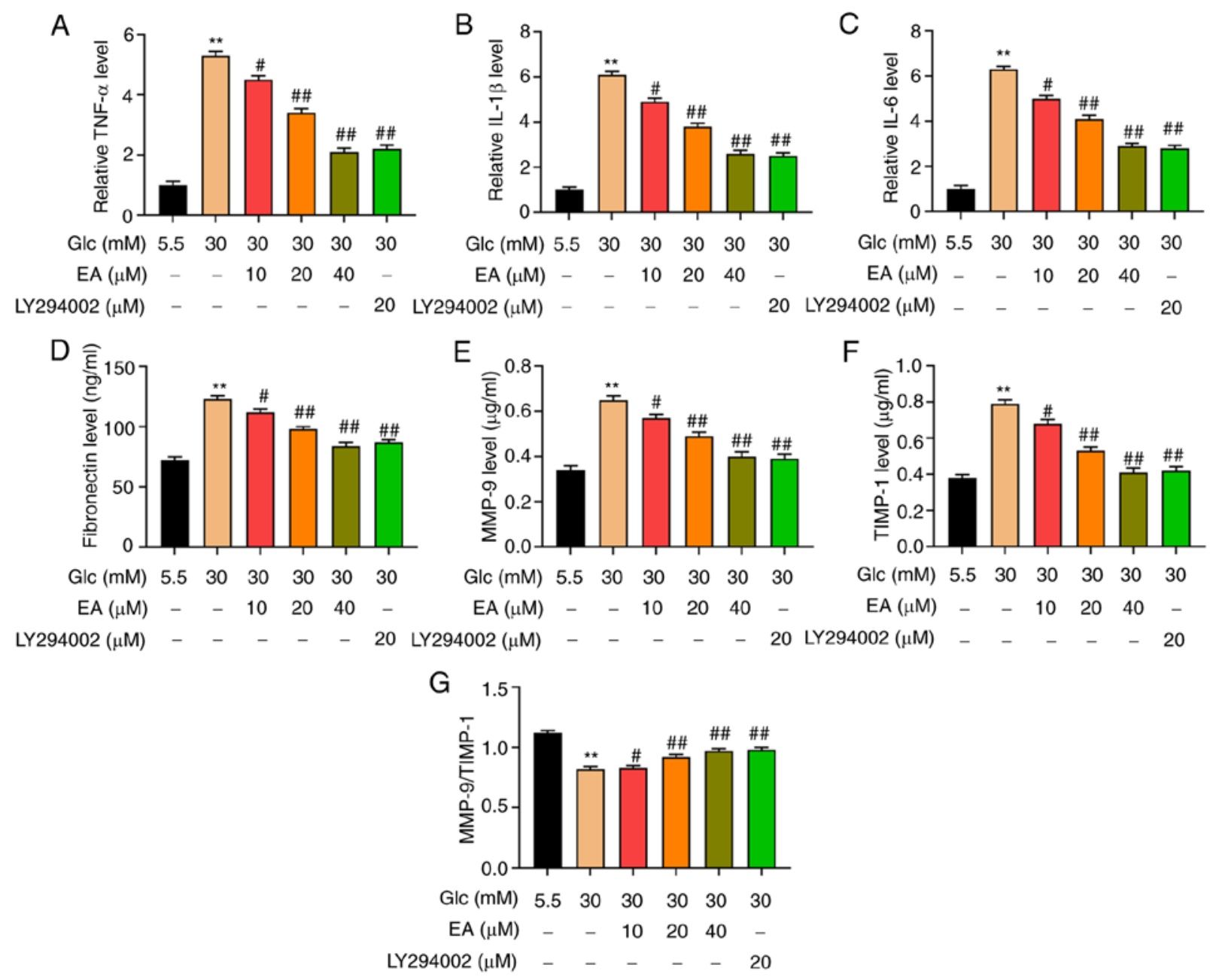

Figure 3. EA inhibits the secretion of inflammatory factors and the production of ECM in MCs induced by HG. Effect of EA (10, 20 and $40 \mu \mathrm{M})$ and LY294002 $(20 \mu \mathrm{M})$ on inflammatory factors (A) TNF- $\alpha$, (B) IL-1 $\beta$ and (C) IL- 6 was detected in MCs induced by HG (30 mM). Effect of EA (10, 20 and $40 \mu \mathrm{M})$ and LY294002 $(20 \mu \mathrm{M})$ on ECM components (D) fibronectin, (E) MMP-9 and (F) TIMP-1 was detected in MCs induced by HG (30 mM). (G) MMP-9/TIMP-1 ratio was analyzed. ${ }^{* * *} \mathrm{P}<0.01$ vs. NC group; ${ }^{*} \mathrm{P}<0.05$ vs. and ${ }^{\# \#} \mathrm{P}<0.01$ vs. HG group. NC group refers to $5.5 \mathrm{mM}$ glucose treated cells. EA, ellagic acid; ECM, extracellular matrix; MCs, mesangial cells; HG, high glucose; Glc, glucose; TIMP-1, tissue inhibitor of metalloproteinases 1.

previous studies, as the MTT and EdU assays indicated that EA could inhibit the proliferation of MCs induced by HG in a concentration-dependent manner. In addition, LY294002 also significantly inhibited cell proliferation induced by HG, indicating that blocking the activation of the PI3K/Akt signaling pathway was an effective way to inhibit the proliferation of MCs.

Oxidative stress plays a notable role in the occurrence and development of $\mathrm{DN}$ (36). HG can increase the production of ROS or decrease the activity of antioxidant enzymes and thus increase the production of MDA (37). It has been previously demonstrated that EA can reduce intracellular oxidative stress (38). In DN, the levels of ROS are notably increased and non-enzymatic antioxidant molecules (glutathione) and antioxidant enzyme (catalase and SOD) activities are decreased (39). Excessive ROS production can activate intracellular signal transduction systems, such as PI3K/Akt, which activate transcription factors, induce ECM protein synthesis and reduce degradation, thereby leading to further development of DN $(36,40)$. EA has been indicated to decrease oxidative stress levels in ischemic-reperfusion injury of renal tissues and cells and inhibit NOX4 expression and phosphorylation of JAK1, JAK2 and STAT1 (41). EA can also reduce the damage of vascular endothelial cells caused by diabetes-induced ROS and function as a free radical scavenger by inhibiting the ERK1/2/NOX4 signaling pathway (42). Similarly, the results of the present study revealed that EA could significantly reduce ROS production, improve SOD activity and decrease the MDA level in MCs treated with HG in a concentration-dependent manner. LY294002, as a specific inhibitor of the PI3K/Akt signaling pathway, could also increase the activity of SOD and decrease the MDA level, thus reducing the levels of ROS.

In DN, the balance of ECM secretion and degradation is disrupted, which leads to the accumulation of ECM (43). DN also causes chronic inflammation, as it has been demonstrated that the secretion of inflammatory factors in MCs is associated with the production of ROS and accumulation of ECM (44). The MMP-9/TIMP-1 system serves a notable role in the degradation of ECM, as it is the main enzyme system of ECM degradation (45). As a representative components of ECM, FN is associated with the adhesion, migration and tissue repair mediated by inflammatory cells in the kidney and also participates in vascular basement membrane thickening and glomerulosclerosis (46). DN also causes chronic inflammation, 
A
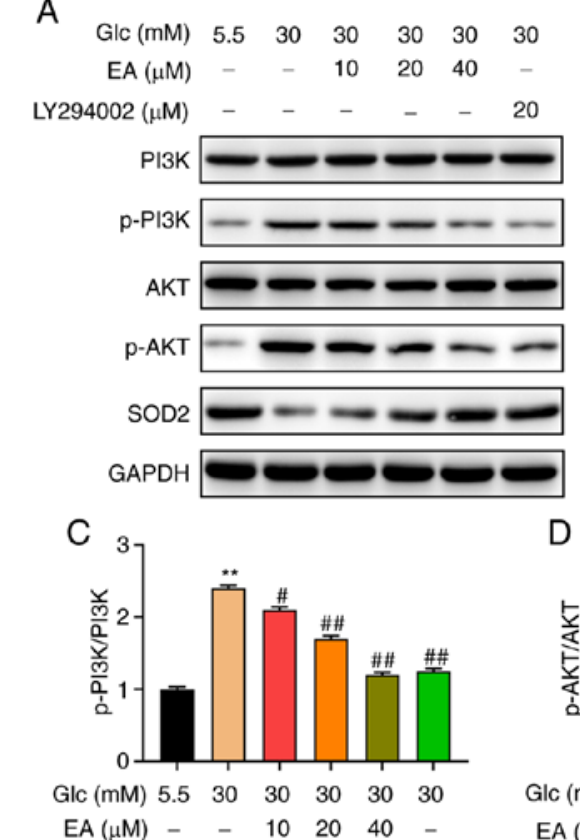

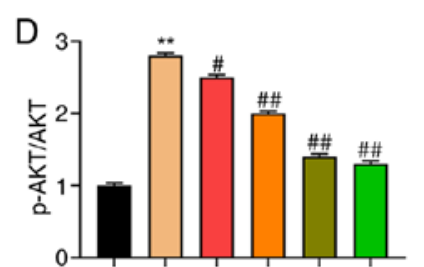

Glc (mM) $\begin{array}{llllll}5.5 & 30 & 30 & 30 & 30 & 30\end{array}$ $\mathrm{EA}(\mu \mathrm{M})$ - $\quad-\begin{array}{llll}10 & 20 & 40 & -\end{array}$
B

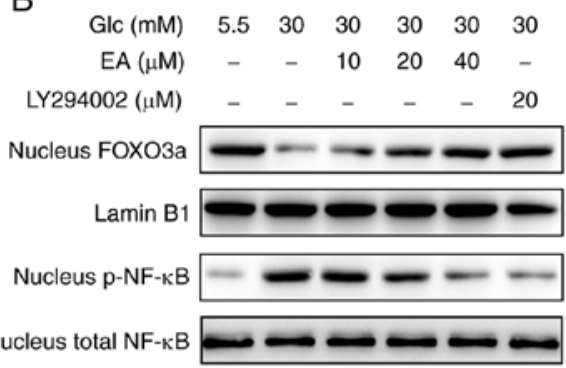
LY294002 $(\mu \mathrm{M})$
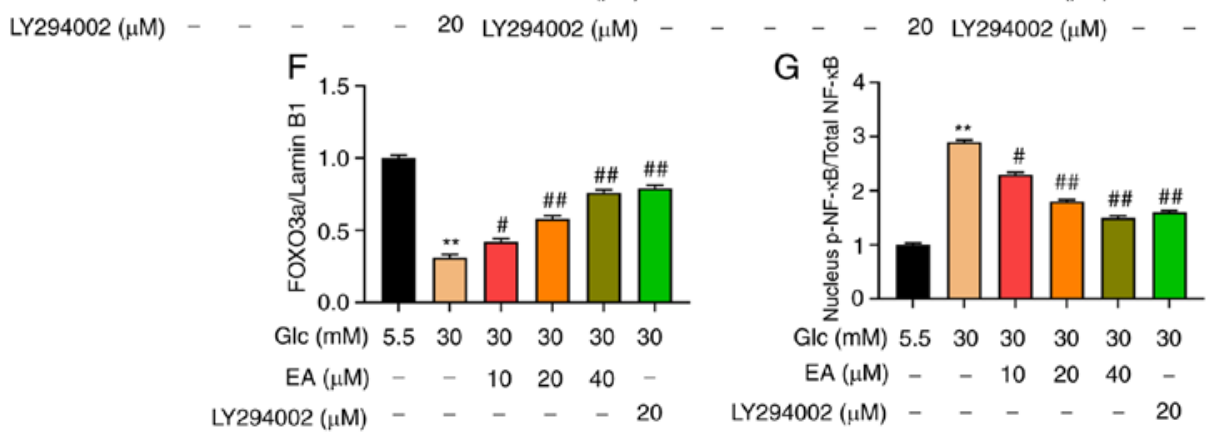

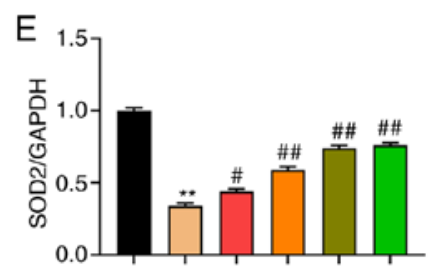

Glc (mM) $\begin{array}{llllll}5.5 & 30 & 30 & 30 & 30 & 30\end{array}$

$\mathrm{EA}(\mu \mathrm{M})$ - - $\begin{array}{llll}10 & 20 \quad 40 \quad-\end{array}$

Figure 4. EA inhibits the PI3K/Akt signaling pathway and regulates its downstream transcription factors in MCs induced by HG. (A) In the cytoplasm, western blotting was used to detect and quantify (C) p-PI3K, (D) p-Akt and (E) SOD2 expression levels in MCs treated with HG (30 mM). (B) Western blotting was used to detect and quantify (F) FOXO3a and (G) p-NF- $\kappa$ B expression levels in the nucleus of MCs treated with $\mathrm{HG}(30 \mathrm{mM}){ }^{* *} \mathrm{P}<0.01$ vs. normal control group; ${ }^{\#} \mathrm{P}<0.05$ and ${ }^{\# \#} \mathrm{P}<0.01$ vs. HG group. NC group refers to $5.5 \mathrm{mM}$ glucose treated cells. HG, high glucose; EA, ellagic acid; MCs, mesangial cells; SOD2, superoxide dismutase 2; Glc, glucose; p-, phosphorylated.

as it has been demonstrated that the secretion of inflammatory factors in MCs is associated with the production of ROS and accumulation of ECM (44). In the present study, the results demonstrated that HGcould significantly increase the expression of FN, MMP-9, TIMP-1, IL-1 $\beta$, IL- 6 and TNF- $\alpha$, but the ratio of MMP-9/TIMP-1 decreased significantly. Moreover, EA and LY294002 could effectively inhibit the HG-induced excessive secretion of FN, MMP-9, TIMP-1, IL-1 $\beta$, IL- 6 and TNF- $\alpha$ and partially reverse the MMP-9/TIMP-1 ratio to normal levels. Previous studies indicated that EA improves renal injury in diabetic rats by inhibiting $\mathrm{NF}-\kappa \mathrm{B}$-induced inflammation (15). EA can also reduce inflammatory factors in other disease models. For instance, EA as an anti-inflammatory factor can improve elastase-induced pneumonia and heart injury in a rat emphysema model (47).

$\mathrm{PI} 3 \mathrm{~K}$ is a mediator between the upstream and downstream signaling molecules activated by growth factor receptor and G-protein-coupled receptor superfamily. PI3K can be activated by a variety of extracellular signals (fibroblast growth factor, vascular endothelial growth factor and Human growth factor). Activated PI3K can promote phosphorylation of Akt, which acts as the second messenger to mediate various cell functions (48).
EA has been indicated to target the PI3K signaling pathway, thus inhibiting the metastasis and proliferation of endothelial cancer cells (49). Cell proliferation and the PI3K/Akt signaling pathway are suppressed by EA in non-small cell lung cancer (50). The dysfunction of MCs induced by HG is associated with the activation of the PI3K/Akt signaling pathway (51). The present study investigated the effect of EA on the PI3K/Akt signaling pathway in MCs induced by HG. Similarly, EA inhibited the phosphorylation of PI3K and Akt. FOXO3a is mainly distributed in mammalian nuclei. Phosphorylated Akt can induce the translocation of FOXO3a from the nucleus to the cytoplasm, thereby inactivating FOXO3a (48). A previous study has demonstrated that upregulation of FOXO3a transcriptional level can increase the expression of SOD2, thus reducing the production of ROS (52). The current study investigated whether EA could regulate intranuclear FOXO3a and SOD2 expression levels. The results revealed that EA treatment significantly increased intranuclear FOXO3a and upregulated the expression level of SOD2 following $\mathrm{HG}$ treatment. $\mathrm{NF}-\kappa \mathrm{B}$, a ubiquitous transcriptional regulator, is a key nuclear factor involved in the regulation of cell proliferation and nuclear inflammation-associated gene 


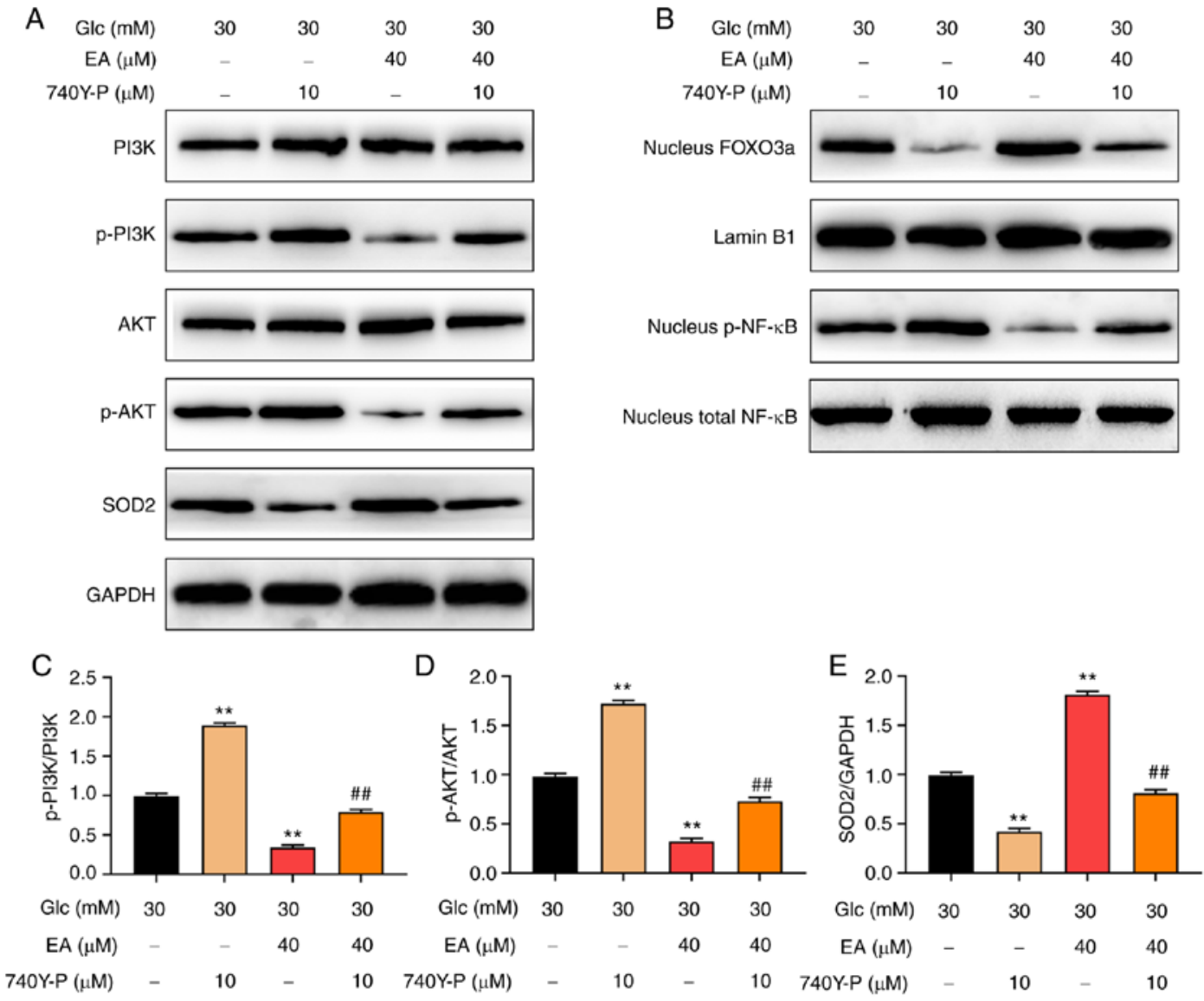

$\mathrm{F}$

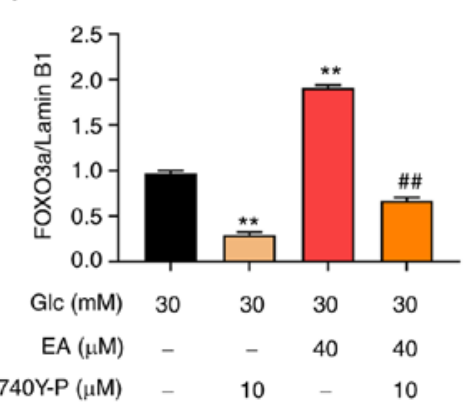

G

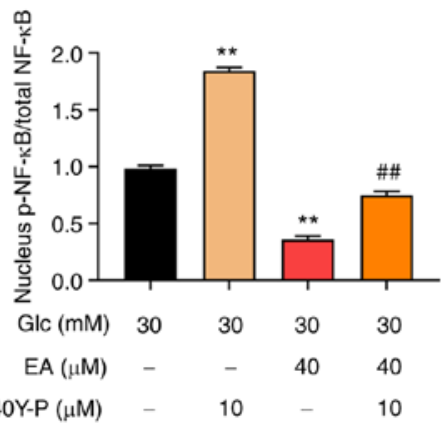

Figure 5. PI3K agonist 740Y-P reverses the effect of EA on the PI3K/Akt signaling pathway and its downstream transcription factors in MCs treated with HG. (A) Western blotting was used to examine the effect of EA (40 $\mu \mathrm{M})$ and/or 740Y-P (10 $\mu \mathrm{M})$ treatment on (C) p-PI3K, (D) p-Akt and (E) SOD2 expression levels in MCs treated with HG $(30 \mathrm{mM})$. (B) Western blotting was used to examine the effect of EA $(40 \mu \mathrm{M})$ and/or $740 \mathrm{Y}-\mathrm{P}(10 \mu \mathrm{M})$ treatment on intranuclear (F) p-NF- $\kappa$ B and (G) FOXO3a in MCs treated with HG (30 mM). ${ }^{* *} \mathrm{P}<0.01$ vs. HG group; ${ }^{\# \# P<0.01 ~ v s . ~ H G ~+~ E A ~ g r o u p . ~ H G, ~ h i g h ~ g l u c o s e ; ~ M C s, ~ m e s a n g i a l ~ c e l l s ; ~}$ p-, phosphorylated; Glc, glucose; EA, ellagic acid; SOD2, superoxide dismutase 2.

transcription. Activation of Akt can promote the transcriptional activity of NF- $\kappa \mathrm{B}(53,54)$. EA improved liver injury in Wistar albino rats and inhibited $\mathrm{NF}-\kappa \mathrm{B}$ expression (55). EA also inhibited $\mathrm{NF}-\kappa \mathrm{B}$ and reduced renal tissue damage in diabetic nephropathy (15). Accordingly, the present study investigated the effect of EA on $\mathrm{NF}-\kappa \mathrm{B}$ and indicated that EA attenuated the phosphorylation of intranuclear NF- $\kappa \mathrm{B}$ in MCs induced by HG. Furthermore, it was examined whether EA attenuated the $\mathrm{MC}$ damage induced by $\mathrm{HG}$ via the PI3K/Akt signaling pathway. The results indicated that the PI3K inhibitor LY294002 exerted the same effect as EA on MCs following HG treatment and the PI3K agonist 740Y-P reversed the effect of EA on the PI3K/Akt signaling pathway and the nuclear expression of $\mathrm{p}-\mathrm{NF}-\kappa \mathrm{B}$ and FOXO3a.
In conclusion, the results of the present study indicated that EA inhibited the activation of the PI3K/Akt signaling pathway in MCs induced by HG and further regulated the transcriptional activities of the downstream transcription factors $\mathrm{FOXO} 3$ and $\mathrm{NF}-\kappa \mathrm{B}$ in the nucleus, thereby inhibiting cell proliferation, oxidative stress, secretion of inflammatory factors and ECM production. The current study provided an experimental theoretical basis on the mechanism via which EA improved the injury of MCs induced by HG. Limitations of the present study were that it did not study the effect of PI3K agonist on the protective effect of EA on MCs induced by high glucose and in vivo experiments on EA improvement of DN were not performed. This is where research could investigate further. 


\section{Acknowledgements}

Not applicable.

\section{Funding}

The present study was supported by Foundation for Innovative Scientific Research of Young and Middle-Aged People in The Second Affiliated Hospital of Harbin Medical University (grant no. KYCX2018-16) and Heilongjiang Postdoctoral Fund (grant no. LBH-Z18128).

\section{Availability of data and materials}

The datasets used and/or analyzed during the current study are available from the corresponding author on reasonable request.

\section{Authors' contributions}

WL and GL contributed to study design, data collection, statistical analysis, data interpretation and manuscript preparation. XK, PG, YS, RD, XW, LC and RY contributed to data collection and statistical analysis. QZ and FK contributed to study design, data interpretation and manuscript preparation. WL and GL confirm the authenticity of all the raw data. All authors reviewed and approved the final manuscript.

\section{Ethics approval and consent to participate}

Not applicable.

\section{Patient consent for publication}

Not applicable.

\section{Competing interests}

The authors declare that they have no competing interests.

\section{References}

1. Ilyas Z, Chaiban JT and Krikorian A: Novel insights into the pathophysiology and clinical aspects of diabetic nephropathy. Rev Endocr Metab Disord 18: 21-28, 2017.

2. Qi C, Mao X, Zhang Z and Wu H: Classification and differential diagnosis of diabetic nephropathy. J Diabetes Res 2017: 8637138, 2017.

3. Wang G, Ouyang J, Li S, Wang H, Lian B, Liu Z and Xie L: The analysis of risk factors for diabetic nephropathy progression and the construction of a prognostic database for chronic kidney diseases. J Transl Med 17: 264, 2019.

4. Furuichi K, Shimizu M, Okada H, Narita I and Wada T: Clinico-pathological features of kidney disease in diabetic cases. Clin Exp Nephrol 22: 1046-1051, 2018.

5. Tung CW, Hsu YC, Shih YH, Chang PJ and Lin CL: Glomerular mesangial cell and podocyte injuries in diabetic nephropathy. Nephrology (Carlton) 23 (Suppl 4): 32-37, 2018.

6. Qiao S, Liu R, Lv C, Miao Y, Yue M, Tao Y, Wei Z, Xia Y and Dai Y: Bergenin impedes the generation of extracellular matrix in glomerular mesangial cells and ameliorates diabetic nephropathy in mice by inhibiting oxidative stress via the mTOR/ $\beta$-TrcP/Nrf2 pathway. Free Radic Biol Med 145 $118-135,2019$.

7. Xu K, Guo L, Bu H and Wang H: Daphnetin inhibits high glucose-induced extracellular matrix accumulation, oxidative stress and inflammation in human glomerular mesangial cells. J Pharmacol Sci 139: 91-97, 2019.
8. Jiang W, Wang R, Liu D, Zuo M, Zhao C, Zhang T and Li W: Protective effects of kaempferitrin on advanced glycation end products induce mesangial cell apoptosis and oxidative stress. Int J Mol Sci 19: 19, 2018.

9. Cui FQ, Wang YF, Gao YB, Meng Y, Cai Z, Shen C, Liu ZQ, Jiang XC and Zhao WJ: Effects of BSF on podocyte apoptosis via regulating the ROS-mediated PI3K/AKT pathway in DN. J Diabetes Res 2019: 9512406, 2019.

10. Manabe E, Handa O, Naito Y, Mizushima K, Akagiri S, Adachi S, Takagi T, Kokura S, Maoka T and Yoshikawa T: Astaxanthin protects mesangial cells from hyperglycemia-induced oxidative signaling. J Cell Biochem 103: 1925-1937, 2008.

11. Moon JY,Tanimoto M,Gohda T, Hagiwara S, Yamazaki T, Ohara I, Murakoshi M, Aoki T, Ishikawa Y, Lee SH, et al: Attenuating effect of angiotensin-(1-7) on angiotensin II-mediated NAD(P)H oxidase activation in type 2 diabetic nephropathy of KK-A(y)/Ta mice. Am J Physiol Renal Physiol 300: F1271-F1282, 2011.

12. Derosa G, Maffioli P and Sahebkar A: Ellagic acid and its role in chronic diseases. Adv Exp Med Biol 928: 473-479, 2016.

13. Zeb A: Ellagic acid in suppressing in vivo and in vitro oxidative stresses. Mol Cell Biochem 448: 27-41, 2018.

14. Ceci C, Lacal PM, Tentori L, De Martino MG, Miano R and Graziani G: Experimental evidence of the antitumor, antimetastatic and antiangiogenic activity of ellagic acid. Nutrients 10: 10, 2018

15. Ahad A, Ganai AA, Mujeeb M and Siddiqui WA: Ellagic acid, an NF- $\mathrm{B}$ B inhibitor, ameliorates renal function in experimental diabetic nephropathy. Chem Biol Interact 219: 64-75, 2014.

16. Lee WJ, Ou HC, Hsu WC, Chou MM, Tseng JJ, Hsu SL, Tsai KL and Sheu WH: Ellagic acid inhibits oxidized LDL-mediated LOX-1 expression, ROS generation, and inflammation in human endothelial cells. J Vasc Surg 52: 1290-1300, 2010.

17. Liu B, Lin J, Bai L, Zhou Y, Lu R, Zhang P, Chen D, Li H, Song J, Liu X, et al: Paeoniflorin inhibits mesangial cell proliferation and inflammatory response in rats with mesangial proliferative glomerulonephritis through PI3K/AKT/GSK-3 $\beta$ pathway. Front Pharmacol 10: 978, 2019.

18. Li F, Zhang Z, Wang P, Wen P, Xu Q, Wang Y, Pan P and Ma L: ALC1 knockdown enhances cisplatin cytotoxicity of esophageal squamous cell carcinoma cells by inhibition of glycolysis through PI3K/Akt pathway. Life Sci 232: 116679, 2019.

19. Zhao C, Gu Y, Chen L and Su X: Upregulation of FoxO3a expression through PI3K/Akt pathway attenuates the progression of lupus nephritis in MRL/lpr mice. Int Immunopharmacol 89 (Pt A): 107027, 2020.

20. Zhang J, Wang X, Vikash V, Ye Q, Wu D, Liu Y and Dong W: ROS and ROS-mediated cellular signaling. Oxid Med Cell Longev 2016: 4350965, 2016.

21. Zhao JH: Mesangial cells and renal fibrosis. Adv Exp Med Biol 1165: 165-194, 2019.

22. Migliorini A, Ebid R, Scherbaum CR and Anders HJ: The danger control concept in kidney disease: Mesangial cells. J Nephrol 26: 437-449, 2013

23. Cao A, Wang L, Chen X, Guo H, Chu S, Zhang X and Peng W: Ursodeoxycholic acid ameliorated diabetic nephropathy by attenuating hyperglycemia-mediated oxidative stress. Biol Pharm Bull 39: 1300-1308, 2016.

24. Dong Z, Sun Y, Wei G, Li S and Zhao Z: Ergosterol ameliorates diabetic nephropathy by attenuating mesangial cell proliferation and extracellular matrix deposition via the TGF- $\beta 1 / \mathrm{Smad} 2$ signaling pathway. Nutrients 11: 11, 2019.

25. Das F, Maity S, Ghosh-Choudhury N, Kasinath BS and Ghosh Choudhury G: Deacetylation of S6 kinase promotes high glucose-induced glomerular mesangial cell hypertrophy and matrix protein accumulation. J Biol Chem 294: 9440-9460, 2019.

26. Lu X, Fan Q, Xu L, Li L, Yue Y, Xu Y, Su Y, Zhang D and Wang L: Ursolic acid attenuates diabetic mesangial cell injury through the up-regulation of autophagy via miRNA-21/PTEN/Akt/mTOR suppression. PLoS One 10: e0117400, 2015.

27. Tan YH, Shudo T, Yoshida T, Sugiyama Y, Si JY, Tsukano C, Takemoto Y and Kakizuka A: Ellagic acid, extracted from Sanguisorba officinalis, induces G1 arrest by modulating PTEN activity in B16F10 melanoma cells. Genes Cells 24: 688-704, 2019.

28. Peng R, Wu Q, Chen J, Ghosh R and Chen X: Isolation of ellagic acid from pomegranate peel extract by hydrophobic interaction chromatography using graphene oxide grafted cotton fiber adsorbent. J Sep Sci 41: 747-755, 2018.

29. Baradaran Rahimi V, Ghadiri M, Ramezani M and Askari VR: Antiinflammatory and anti-cancer activities of pomegranate and its constituent, ellagic acid: Evidence from cellular, animal, and clinical studies. Phytother Res 34: 685-720, 2020. 
30. Huang Q, Chai WM, Ma ZY, Deng WL, Wei QM, Song S, Zou ZR and Peng YY: Antityrosinase mechanism of ellagic acid in vitro and its effect on mouse melanoma cells. J Food Biochem 43: e12996, 2019.

31. Cove-Smith A and Hendry BM: The regulation of mesangial cell proliferation. Nephron Exp Nephrol 108: e74-e79, 2008

32. Kurogi Y: Mesangial cell proliferation inhibitors for the treatment of proliferative glomerular disease. Med Res Rev 23: 15-31, 2003

33. Wolf G: Molecular mechanisms of diabetic mesangial cell hypertrophy: A proliferation of novel factors. J Am Soc Nephrol 13: 2611-2613, 2002.

34. Yousuf M, Shamsi A, Khan P, Shahbaaz M, AlAjmi MF Hussain A, Hassan GM, Islam A, Rizwanul Haque QM and Hassan MI: Ellagic acid controls cell proliferation and induces apoptosis in breast cancer cells via inhibition of cyclin-dependent kinase 6. Int J Mol Sci 21: 21, 2020.

35. Malik A, Afaq S, Shahid M, Akhtar K and Assiri A: Influence of ellagic acid on prostate cancer cell proliferation: A caspase-dependent pathway. Asian Pac J Trop Med 4: 550-555, 2011.

36. Jha JC, Banal C, Chow BS, Cooper ME and Jandeleit-Dahm K Diabetes and kidney disease: Role of oxidative stress. Antioxid Redox Signal 25: 657-684, 2016.

37. Sztanek F, Molnárné Molnár Á and Balogh Z: The role of oxidative stress in the development of diabetic neuropathy. Orv Hetil 157: 1939-1946, 2016 (In Hu).

38. Zhou B, Li Q, Wang J, Chen P and Jiang S: Ellagic acid attenuates streptozocin induced diabetic nephropathy via the regulation of oxidative stress and inflammatory signaling. Food Chem Toxicol 123: 16-27, 2019.

39. Giacco F and Brownlee M: Oxidative stress and diabetic complications. Circ Res 107: 1058-1070, 2010.

40. Kashihara N, Haruna Y, Kondeti VK and Kanwar YS: Oxidative stress in diabetic nephropathy. Curr Med Chem 17: 4256-4269, 2010.

41. Liu Q, Liang X, Liang M, Qin R, Qin F and Wang X: Ellagic acid ameliorates renal ischemic-reperfusion injury through NOX4/JAK/STAT signaling pathway. Inflammation 43: 298-309, 2020 .

42. Rozentsvit A, Vinokur K, Samuel S, Li Y, Gerdes AM and Carrillo-Sepulveda MA: Ellagic acid reduces high glucose induced vascular oxidative stress through ERK1/2/NOX4 Signaling Pathway. Cell Physiol Biochem 44: 1174-1187, 2017.

43. Kolset SO, Reinholt FP and Jenssen T: Diabetic nephropathy and extracellular matrix. J Histochem Cytochem 60: 976-986, 2012.

44. Wada $\mathrm{J}$ and Makino $\mathrm{H}$ : Inflammation and the pathogenesis of diabetic nephropathy. Clin Sci (Lond) 124: 139-152, 2013.
45. Rysz J, Banach M, Stolarek RA, Pasnik J, Cialkowska-Rysz A, Koktysz R, Piechota $M$ and Baj Z: Serum matrix metalloproteinases MMP-2 and MMP-9 and metalloproteinase tissue inhibitors TIMP-1 and TIMP-2 in diabetic nephropathy. J Nephrol 20: 444-452, 2007.

46. Hu C, Sun L, Xiao L, Han Y, Fu X, Xiong X, Xu X, Liu Y, Yang S, Liu F, et al: Insights into the mechanisms involved in the expression and regulation of extracellular matrix proteins in diabetic nephropathy. Curr Med Chem 22: 2858-2870, 2015.

47. Mansouri Z, Dianat M, Radan M and Badavi M: Ellagic acid ameliorates lung inflammation and heart oxidative stress in elastase-induced emphysema model in rat. Inflammation 43: 1143-1156, 2020.

48. Zhang $\mathrm{M}$ and Zhang $\mathrm{X}$ : The role of PI3K/AKT/FOXO signaling in psoriasis. Arch Dermatol Res 311: 83-91, 2019.

49. Wang Y, Ren F, Li B, Song Z, Chen P and Ouyang L: Ellagic acid exerts antitumor effects via the PI3K signaling pathway in endometrial cancer. J Cancer 10: 3303-3314, 2019.

50. Liu Q, Liang X, Niu C and Wang X: Ellagic acid promotes A549 cell apoptosis via regulating the phosphoinositide 3-kinase/protein kinase B pathway. Exp Ther Med 16: 347-352, 2018.

51. Wang EM, Fan QL, Yue Y and Xu L: Ursolic acid attenuates high glucose-mediated mesangial cell injury by inhibiting the phosphatidylinositol 3-kinase/Akt/mammalian target of rapamycin (PI3K/Akt/mTOR) signaling pathway. Med Sci Monit 24: 846-854, 2018

52. Kops GJ, Dansen TB, Polderman PE, Saarloos I, Wirtz KW, Coffer PJ, Huang TT, Bos JL, Medema RH and Burgering BM: Forkhead transcription factor FOXO3a protects quiescent cells from oxidative stress. Nature 419: 316-321, 2002.

53. Rangan G, Wang Y and Harris D: NF-kappaB signalling in chronic kidney disease. Front Biosci 14: 3496-3522, 2009.

54. Yang L, Hu X and Mo YY: Acidosis promotes tumorigenesis by activating $\mathrm{AKT} / \mathrm{NF}-\kappa \mathrm{B}$ signaling. Cancer Metastasis Rev 38: 179-188, 2019.

55. Aslan A, Gok O, Erman O and Kuloglu T: Ellagic acid impedes carbontetrachloride induced liver damage in rats through suppression of $\mathrm{NF} \mathrm{kB,} \mathrm{Bcl} 2$ and regulating $\mathrm{Nrf} 2$ and caspase pathway. Biomed Pharmacother 105: 662-669, 2018.

(i) (2) This work is licensed under a Creative Commons Attribution-NonCommercial-NoDerivatives 4.0 International (CC BY-NC-ND 4.0) License. 